\title{
The comparative effectiveness of ventricular shunt placement versus endoscopic third ventriculostomy for initial treatment of hydrocephalus in infants
}

\author{
Clinical article
}

\author{
Sarah C. Jernigan, M.D., M.P.H., ${ }^{1}$ Jay G. Berry, M.D., M.P.H., ${ }^{2}$ \\ Dionne A. Graham, Ph.D., ${ }^{3}$ and Liliana Goumnerova, M.D. 1 \\ ${ }^{1}$ Department of Neurosurgery, ${ }^{2}$ Division of General Pediatrics, and ${ }^{3}$ Clinical Research Program, Boston \\ Children's Hospital, Harvard Medical School, Boston, Massachusetts
}

\begin{abstract}
Object. The purpose of this study was to compare the effectiveness of CSF diversion with endoscopic third ventriculostomy (ETV) versus shunt therapy in infants with hydrocephalus.

Methods. The authors conducted a retrospective analysis of 5416 infants 1 year of age or younger with hydrocephalus (congenital or acquired) in whom CSF diversion was performed using either ETV or shunt placement at 41 children's hospitals between 2004 and 2009. Data were obtained from the Pediatric Health Information Systems database. Surgical failure was defined as the need for a repeat diversion operation within 1 year of initial surgery. The authors compared failure rates of ETV and shunt, as well as patient demographics and clinical characteristics, using hierarchical regression according to treatment group.

Results. During the period examined, 872 infants (16.1\%) initially underwent ETV and 4544 (83.9\%) underwent ventricular shunt placement. The median infant age was 37 days (IQR 11-122 days) for both ETV and shunt placement. More infants who underwent ETV rather than shunt placement were born prematurely $(41.6 \%$ vs $23.9 \%$, respectively; $\mathrm{p}<0.01)$ and had intraventricular hemorrhage ( $45.4 \%$ vs $17.5 \%$, respectively; $\mathrm{p}<0.01)$. Higher operative failure rates at 1 year were observed in infants who underwent ETV as opposed to shunt surgery (64.5\% vs $39.6 \%$, respectively; OR 2.9 [95\% CI 2.3-3.5], p < 0.01). After controlling for prematurity, intraventricular hemorrhage, and spina bifida, ETV remained associated with a higher risk of failure (OR 2.6 [95\% CI 2.1-3.2]).

Conclusions. In infants with hydrocephalus, a greater 1-year CSF diversion failure rate may occur after ETV compared with shunt placement. This risk is most significant for procedures performed within the first 90 days of life. Further investigation of the need for multiple reoperations, cost, and impact of surgeon and hospital experience is necessary to distinguish which treatment is more effective in the long term.

(http://thejns.org/doi/abs/10.3171/2013.11.PEDS13138)
\end{abstract}

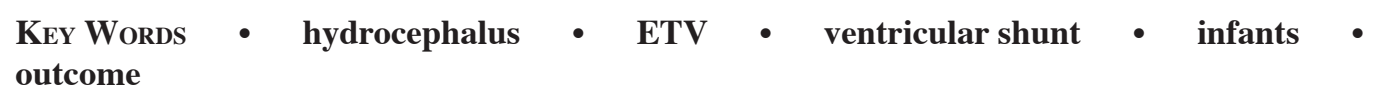

$\mathrm{C}$ EREBROSPINAL fluid diversion for treatment of hydrocephalus is one of the most common pediatric neurosurgery procedures performed. The annual hospital cost of treating children with hydrocephalus is approximately $\$ 2$ billion. ${ }^{22}$

Cerebrospinal fluid diversion through a shunt catheter remains the preferred treatment method in hydrocephalus. Despite the development of new shunt materials and techniques for introduction of catheters, ${ }^{1,3}$ however, the failure rate associated with a shunt within the 1st year has remained high since 1960, ranging from $25.7 \%$ to $36.8 \%$ in the year $2000 .{ }^{4}$ Shunt failure represents a significant health care expense, with an estimated cost of over $\$ 1$

\footnotetext{
Abbreviations used in this paper: $\mathrm{CPC}=$ choroid plexus cauterization; ETV = endoscopic third ventriculostomy; IVH = intraventricular hemorrhage; PHIS = Pediatric Health Information System ; $\mathrm{VP}=$ ventriculoperitoneal .
}

billion dollars annually for admissions for both pediatric and adult patients. ${ }^{18}$ It is believed that reducing the failure rate of CSF diversion within 1 year of treatment could lead to substantial improvement of the health of children with hydrocephalus and the hefty attendant health care expenditures. ${ }^{24}$

Younger patients, specifically infants under 1 year of age at the time of initial shunt placement, have the highest risk of shunt failure. ${ }^{17,19}$ Alternative and potentially more successful methods to divert CSF in infants with hydrocephalus are being explored. Endoscopic third ventriculostomy (ETV), the original hydrocephalus treatment modality from the early 20th century, has regained attention in recent years as an alternative to catheter-shunted CSF diversion. However, single-institutional studies re-

This article contains some figures that are displayed in color online but in black-and-white in the print edition. 
port that ETV is also associated with high failure rates (range 70\%-80\%) within 1 year in high-risk patients, such as infants under 1 year of age. ${ }^{10-13,18}$ Therefore, we compared the effectiveness of ETV and shunt placement for hydrocephalus treatment in a multicenter cohort of infants younger than 1 year.

\section{Methods}

Study Design and Setting

This study was a retrospective cohort analysis of the Pediatric Health Information System (PHIS), an administrative database of 39 freestanding (financially independent), not-for-profit, tertiary care pediatric hospitals in the United States. ${ }^{12}$ These hospitals are affiliated with the Children's Hospital Association (Shawnee Mission, KS), a business alliance of children's hospitals. The data warehouse function for the PHIS database is managed by Solucient LLC (Evanston, IL). For purposes of external benchmarking, participating hospitals provide discharge data, including demographics, diagnoses, and procedures. Data are subjected to a number of reliability and validity checks and are processed into data quality reports. Coding of the PHIS database permits tracking of subsequent hospitalizations within the same hospital for individual patients using an encrypted medical record number.

\section{Patient Population}

The study population was infants aged less than 1 year with hydrocephalus who underwent CSF diversion via ventricular shunt placement or ETV between January 1, 2004, and December 1, 2009. Infants with hydrocephalus were identified using ICD-9-CM diagnosis codes of congenital hydrocephalus (742.3), communicating hydrocephalus (331.3), obstructive hydrocephalus (331.4), or spina bifida with hydrocephalus (741.0x). ${ }^{1}$

\section{Main Exposure}

The main exposure was initial hydrocephalus treatment with shunt or ETV within the 1st year of life. We examined all hospitalizations dating back to the child's birth to determine the date of the initial treatment.

Shunt. Patients with initial ventricular shunt placement were identified with the ICD-9-CM procedural code of ventricular shunt placement $(02.3 \mathrm{x})$ during a hospital admission. All types of shunts were included for analysis. A ventriculoperitoneal (VP) shunt was defined as "ventricular shunt to abdominal cavity and organs" (02.34), and the "other" shunt type category included "ventricular shunt to circulatory system" (02.32), "ventricular shunt to thoracic cavity" (02.33), "ventricular shunt to urinary system" (02.35), and "other operations to establish drainage of ventricle" (02.39). ${ }^{1}$

Endoscopic Third Ventriculostomy. Patients undergoing ETV for their first hydrocephalus treatment were identified with the ICD-9-CM procedural code for ventriculostomy (02.2) during a hospital admission in the absence of billing charges for implanted ventricular catheters, reservoirs, or shunts suggestive of external ventricular drain placement or ventriculosubgaleal shunt placement. ${ }^{1}$

\section{Main Outcome Measure}

For patients with shunt-treated hydrocephalus, the primary outcome of the study was treatment failure, defined as a revision or replacement of an existing ventricular shunt within 1 year of the initial procedure. Ventricular shunt revisions were identified using the ICD-9-CM procedural codes 1) for ventricular shunt replacement $(02.42,54.95)$ or 2$)$ for ventricular shunt removal $(02.43)$ and new ventricular shunt placement (02.34), or other shunt-related procedure $(02.3 \mathrm{x})$, in a patient with an existing ventricular shunt placed during a hospital admission. Patients undergoing a sequence of shunt removal and temporary ventricular drain placement followed by insertion of a new ventricular shunt were considered to have had a single episode in which an existing shunt was replaced. We assumed that a patient had no revision if there were no hospital admissions for revision. ${ }^{1}$

For patients who had undergone ETV, treatment failure was defined as a subsequent surgical intervention for hydrocephalus, including repeat ETV or shunt placement within 1 year of initial ETV. The secondary outcome of survivability reflects any further surgical interventions for hydrocephalus that occurred at least 1 year after initial shunt placement or ETV but within the overall study period. We assessed data for all hospitalizations within the PHIS network for the entirety of the study period, such that the first patients to be treated had follow-up for up to 5 years after their initial CSF diversion procedure but the last patients had only 1 year of follow-up.

\section{Covariates}

Demographic characteristics assessed at the time of initial shunt placement included primary payer (private insurance, government insurance, or other), age, sex, and race (non-Hispanic Caucasian, non-Hispanic black, Hispanic, Asian, and other). The category "other" primary payer includes individuals who are self-insured. The "other" category of race includes all individuals who were not classified as Caucasian, black, Hispanic, or Asian.

We evaluated clinical characteristics that may be correlated with the likelihood of CSF diversion failure, including prematurity (ICD-9-CM 765.2x), low birth weight $(765.0,765.1)$, myelomeningocele with hydrocephalus (741.0x), intraventricular hemorrhage (IVH; 772.1), or multiple congenital anomalies (743.xx through 759 . $\mathrm{xx})$. We also evaluated hospital characteristics including the annual hospital volume of ETV- and shunt-treated patients, teaching status, and geographic region (Northeast, South, Midwest, and West).

\section{Statistical Analysis}

We assessed unadjusted differences in treatment failure rates for ETV and shunt as well as risk factors for treatment failure with a Rao-Scott chi-square test that accounted for the random effect of individual hospitals. To adjust for case-mix differences, we used generalized estimating equations to assess differences in failure rates for ETV and shunt surgery accounting for demographic, clinical, and hospital characteristics associated $(\mathrm{p}<1.0)$ with treatment failure in the unadjusted analysis, and the random ef- 


\section{Shunt versus ETV for initial treatment of hydrocephalus}

fect of hospitals. We also performed partition, regression tree modeling with binary splits for continuous variables (for example, age in days) to assess which patients had the greatest success and failure with ETV and shunt treatment.

\section{Results}

There were 5416 infants less than 1 year of age with hydrocephalus in the study cohort; 4544 of them (83.9\%) underwent shunt placement and 872 (16.1\%) underwent ETV for the initial treatment of their hydrocephalus. The 1-year failure rates for shunt and ETV treatments were $39.6 \%$ and $64.5 \%$, respectively.

\section{Hospital Characteristics}

The mean annual hospital case volume was significantly greater for shunt placement than for ETV (mean $26.6 \pm 14.0$ cases vs $5.3 \pm 4.9$ cases $[ \pm$ SD], $p<0.01$ ). Neither shunt nor ETV failure was associated with hospital volume, geographic region, or teaching status (all p >0.1) (Table 1, Fig. 1).

\section{Patient Characteristics}

The median infant age was 37 days (IQR 11-122 days) for both ETV and shunt placement. More infants who underwent ETV were born prematurely (41.6\% vs $23.9 \%$, respectively; $\mathrm{p}<0.01)$ and had intraventricular hemorrhage ( $45.4 \%$ vs $17.5 \%$, respectively; $\mathrm{p}<0.01$ ).

In infants less than 90 days of age, we noted significantly higher failure rates for both shunt treatment $(42.4 \%$ vs $30.0 \%, \mathrm{p}<0.001)$ and ETV $(68.6 \%$ vs $54.6 \%, \mathrm{p}=0.01)$ compared with infants 91-365 days of age at initial CSFdiversion surgery respectively. Neither shunt nor ETV failure was influenced by sex, race, or insurance payer (all $\mathrm{p}$ $>0.1$ ). In infants with prematurity/low birth weight, IVH, and myelomeningocele, significantly higher shunt and ETV failure rates were documented (all $\mathrm{p}<0.05$ ). Intraventricular hemorrhage was associated with the highest shunt failure rate $52.6 \%$ in those with IVH compared with $36.7 \%$ in those without IVH; $\mathrm{p}<0.001$ ) and myelomeningocele was associated with the highest ETV failure rate $(77.4 \%$ in those with myelomeningocele compared with $63.5 \%$ without myelomeningocele; $\mathrm{p}=0.03$ ) (Tables 2 and 3 ).

TABLE 1: Hospital characteristics

\begin{tabular}{lc}
\hline \multicolumn{1}{c}{ Hospital Data } & No. of Hospitals (\%) \\
\hline geographic region & $6(15.4)$ \\
northeast & $10(25.6)$ \\
north central & $14(35.9)$ \\
south & $9(23.1)$ \\
west & \\
academic & $22(56.4)$ \\
no & $17(43.6)$ \\
yes & \\
mean annual case vol $( \pm$ SD) & $26.6 \pm 14.0$ \\
shunt & $5.3 \pm 4.9$ \\
ETV &
\end{tabular}
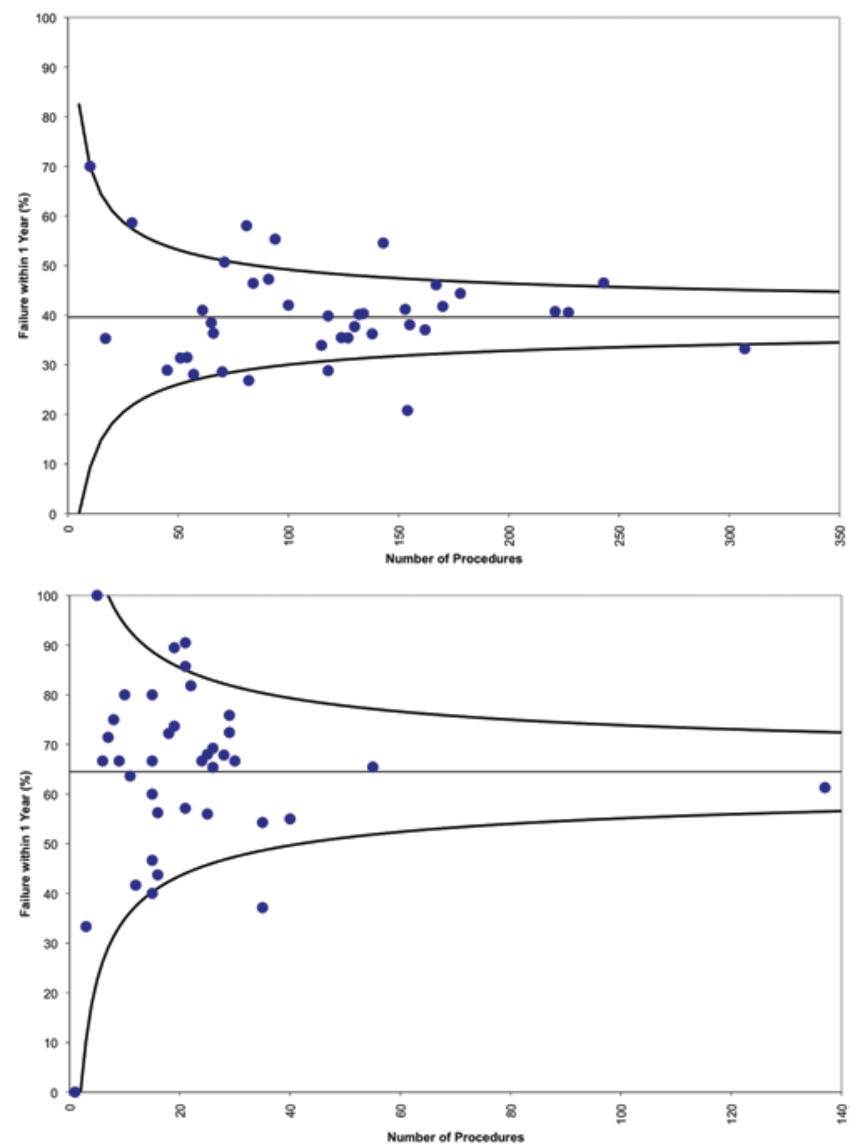

Fig. 1. Failure rate within 1 year as a function of hospital volume of VP shunt (upper) and ETV (lower) procedures in infants.

\section{Comparative Effectiveness of Shunt and ETV}

In multivariate analysis, we documented a significantly increased risk of surgical failure for infants under 1 year treated with ETV compared with shunt placement, with an adjusted odds ratio of 2.6 (95\% CI 2.1-3.2, p < 0.001). For both ETV and shunt treatment, age less than 90 days, prematurity, and IVH remained significantly associated with treatment failure in the multivariate analysis (all p < 0.001) (Table 3). In patients with myelomeningocele, the treatment failure was higher in those who had undergone ETV rather than shunt insertion (OR 1.8 [95\% CI 1.2-2.7], $\mathrm{p}<0.005)$. However, there was no difference in the failure rates between shunts and ETVs in multivariate analysis (unadjusted OR 1.0, 95\% CI 0.9-1.2; adjusted OR 1.1, 95\% CI 1.0-1.4) (Table 4). There was a $100 \%$ failure rate for ETV in the 18 prematurely born infants with a myelomeningocele. For both ETV and shunt treatment, age less than 90 days, prematurity, and IVH remained significantly associated with treatment failure in the multivariate analysis (all p $<0.001$ ). Endoscopic third ventriculostomy was most successful in patients 90 days of age or older regardless of comorbidities (54.6\% failure rate) (Table 4). Patients were followed throughout the time period of the study, and for both shunt and ETV treatments, the highest risk of failure was within the first 90 days after the initial procedure. In patients with 
TABLE 2: Failure rates of ventricular shunt by demographics and comorbidities*

\begin{tabular}{lccc}
\hline \multicolumn{1}{c}{ Shunt Data } & No. of Cases & \% Surgical Failure & $p$ Value \\
\hline age (days) & & & \\
$1-30$ & 2050 & 45.2 & $<0.001$ \\
$31-90$ & 992 & 42.4 & $<0.001$ \\
$91-365$ & 1502 & 30.0 & ref \\
sex & & & \\
male & 2501 & 39.4 & ref \\
female & 2043 & 39.7 & 0.8 \\
race† & & & \\
Caucasian & 3050 & 40.1 & ref \\
African American & 789 & 38.9 & 0.5 \\
Asian & 50 & 44 & 0.6 \\
other & 499 & 39.3 & 0.7 \\
prematurity & & & \\
no & 3428 & 36.5 & ref \\
yes & 1116 & 49.1 & $<0.001$ \\
IVH & & & \\
no & 3732 & 36.7 & ref \\
yes & 812 & 52.6 & $<0.001$ \\
MMC & & & ref \\
no & 3332 & 38.5 & $\mathbf{0 . 0 2}$ \\
yes & 1212 & 42.5 & \\
\hline
\end{tabular}

* Boldface type indicates statistical significance. MMC = myelomeningocele; ref $=$ reference.

† Data missing for 156 patients (surgical failure rate $31.4 \% ; p=0.03$ ).

5 years of follow-up, the shunt group still had a higher percentage of patients free from revision compared with the ETV group, but the long-term success rates for both procedures approached equality at 5 years (Fig. 2).

\section{Discussion}

Outcomes for infants with hydrocephalus treated with ETV and shunts are different. Failure rates for ETV are higher than those for shunt in infants aged 90 days or less and for infants with a myelomeningocele. The highest failure rate for ETV was observed in infants treated within the first 90 days of life and with diagnoses of myelomeningocele and prematurity, whereas ETV was the most successful in infants older than 90 days, regardless of other medical comorbidities.

Success rates for ETV vary from $20 \%$ to $70 \%$ in prior studies of small groups of infants younger than 1 year of age. . $^{70,14}$ In our study, the ETV success decreased from $40 \%-45 \%$ to $25 \%-30 \%$ in infants younger than 90 days of age or with comorbidities. ${ }^{11-13}$ In another study of 11 patients under the age of 1 year who underwent a second endoscopic procedure after failed ETV, the authors found either a completely or almost completely closed stoma or new arachnoid membranes obstructing the stoma in all 11 patients and hypothesized that these young patients are at an increased risk of ETV failure because new arachnoid
TABLE 3: Failure rates of ETV by demographics and comorbidities*

\begin{tabular}{|c|c|c|c|}
\hline ETV Data & No. of Cases & $\%$ Surgical Failure & $p$ Value \\
\hline \multicolumn{4}{|l|}{ age (days) } \\
\hline $1-30$ & 266 & 67.9 & 0.001 \\
\hline $31-90$ & 220 & 70.0 & 0.01 \\
\hline $91-365$ & 260 & 54.6 & ref \\
\hline \multicolumn{4}{|l|}{ sex } \\
\hline male & 520 & 64.4 & ref \\
\hline female & 352 & 64.5 & 0.95 \\
\hline \multicolumn{4}{|l|}{ race† } \\
\hline Caucasian & 521 & 63.5 & ref \\
\hline African American & 192 & 69.8 & 0.1 \\
\hline Asian & 13 & 53.8 & 0.5 \\
\hline other & 123 & 62.6 & 0.9 \\
\hline \multicolumn{4}{|l|}{ prematurity } \\
\hline no & 513 & 59.5 & ref \\
\hline yes & 359 & 71.6 & $<0.001$ \\
\hline \multicolumn{4}{|l|}{ IVH } \\
\hline no & 479 & 61.4 & ref \\
\hline yes & 393 & 68.2 & 0.04 \\
\hline \multicolumn{4}{|l|}{ MMC } \\
\hline no & 810 & 63.5 & ref \\
\hline yes & 62 & 77.4 & 0.03 \\
\hline
\end{tabular}

membranes that block their ETV stomas are more likely to form. ${ }^{26}$ While the risk factors of age less than 3 months, prematurity, and IVH all had increased adjusted odds ratio of failure for ETV compared shunt therapy, the diagnosis of myelomeningocele was not associated with an increased risk of failure of ETV over shunt because patients with myelomeningoceles are at an increased risk of failure of any CSF-diversion procedure compared with other children.

In many studies, age at the time of initial shunt placement has been shown to be an important risk factor for shunt failure in the child's 1st year of life. ${ }^{1,6,15,16,24}$ Each of these studies included all children under the age of 1 year in a single group. We found that children aged 0-30 days, 30-60 days, and 60-90 days had a significantly increased failure rate for both VP shunts and ETVs compared with children 91-365 days of age, regardless of comorbidities, which indicates some change either in the etiology of or compensation to hydrocephalus in infants under 3 months versus those 3-12 months.

Previous studies have shown that the majority of hospitals perform pediatric ventriculoperitoneal shunt operations in relatively low volume, with $75 \%$ of hospitals performing fewer than 20 initial shunt procedures annually., $, 6,12$ While higher hospital volume for surgical procedures has been correlated with better outcomes in a variety of specialties, including neurosurgery, $2,11,20,25$ there have been conflicting data in regard to volume and outcome for ventriculoperitoneal shunts. While 2 prior studies found a 
Shunt versus ETV for initial treatment of hydrocephalus

TABLE 4: Adjusted risk of surgical failure within 1 year*

\begin{tabular}{lcccc}
\hline \multirow{2}{*}{ Factor } & \multicolumn{4}{c}{ Risk of Surgical Failure } \\
\cline { 2 - 5 } & Unadjusted OR $(95 \% \mathrm{Cl})$ & $\mathrm{p}$ Value & Adjusted OR $(95 \% \mathrm{Cl})$ & $\mathrm{p} \mathrm{Value}$ \\
\hline all patients: ETV vs VPS & $2.9(2.3-3.5)$ & $<0.001$ & $2.6(2.1-3.2)$ & $<0.001$ \\
0-90 vs 91-365 days & $1.9(1.7-2.1)$ & $<0.001$ & $1.6(1.4-1.8)$ & $<0.001$ \\
prematurity & $1.8(1.6-2.1)$ & $<0.001$ & $1.3(1.2-1.5)$ & $<0.001$ \\
IVH & $2.1(1.8-2.4)$ & $<0.001$ & $1.4(1.2-1.7)$ & $<0.001$ \\
MMC & $1.0(0.9-1.2)$ & 0.75 & $1.1(1.0-1.4)$ & 0.15 \\
\hline
\end{tabular}

* Boldface type indicates statistical significance. VPS = VP shunt.

decrease in infection rates and mortality rates in highervolume centers, ${ }^{5,21}$ other studies noted no correlation between volume and patient survival ${ }^{13}$ or found that hospital volume only affected revision rate in 2 hospitals whose annual volume was significantly higher than that of the other 30 hospitals, with more than 80 initial shunts placed annually versus fewer than forty placed annually at most other hospitals. ${ }^{1}$ We found no correlation between hospital volume and outcomes for either shunt placement or ETV.

In a recent study Warf et al. compared the success of ETV versus ETV with choroid plexus cauterization (CPC) in infants less than 1 year of age in Uganda. They found that ETV was effective in treating hydrocephalus in only $47 \%$ of patients with myelomeningocele or noninfectious hydrocephalus compared with a success rate of $66 \%$ when ETV was combined with CPC in this same patient population. In the patients with postinfectious hydrocephalus, no statistically significant difference was seen between ETV alone (52\% success) compared with combined ETV-CPC (62\% success), but there was a trend toward significance. There were very few patients with posthemorrhagic hydrocephalus in this African population (only $1 \%$ of all study patients) because very few premature infants with significant posthemorrhagic hydrocephalus survive..$^{27}$ In our study, we were unable to elucidate which patients might have undergone ETV with CPC versus ETV alone since there is no separate coding for these 2 procedures.

Although the present study is limited by its retrospective review of an administrative database, it allowed

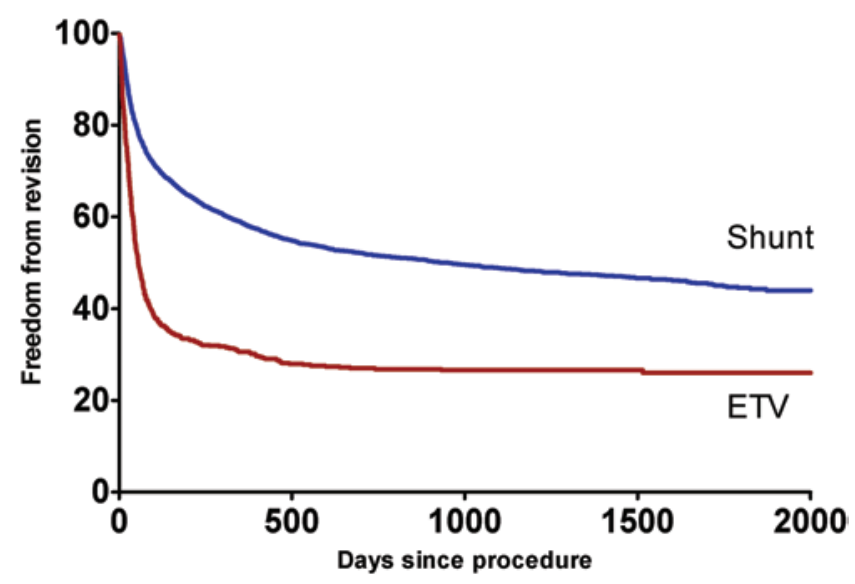

FIG. 2. Kaplan-Meier curve for the 5-year survival of all VP shuntand ETV-treated patients in the study. for a much larger number of patients than prior studies in an attempt to elucidate risk factors in a specific patient population. Coding errors could affect an administrative database, but we internally validated the PHIS patients from Boston Children's Hospital by using our internal departmental database to ensure that our patient collection algorithm was both sensitive and specific. However, we cannot account for patients who underwent a secondary CSF-diversion procedure at an institution outside of the hospitals included in the PHIS consortium, and such patients would be included in our study as not having had a surgical failure. Also, patients who underwent initial shunt placement were not screened for subsequent endoscopic procedures, which could also indicate a potential failure in the initial CSF diversion. The impact of these limitations is lessened by the increased power in the study given the large patient population. One final limitation of utilizing administrative databases is the lack of clinical information available. There is no way of determining other complications of these procedures such as infection, neurological deficit, hematoma, and so on. Finally, our primary outcome of failure was defined as reoperation within 1 year, but our secondary outcome was any additional surgical interventions for hydrocephalus throughout the study period, allowing us to calculate shunt survival for at least a portion of the cohort population for as many as 5 years after their initial procedure.

Our rates of shunt and ETV failure correlate both with published data and the mathematical model developed by Stein and Guo, which estimated a shunt failure rate of $64.2 \%$ at 1 year and $49.4 \%$ at 5 years, with a median survival of 4.9 years. ${ }^{23}$

\section{Conclusions}

This is the first study to evaluate the comparative effectiveness of shunt treatment and ETV in a multiinstitutional cohort of infants with hydrocephalus. Although there are limitations to studies utilizing administrative databases, the large patient populations that they allow to be studied enable an analysis of a wide variety of risk factors and patient characteristics that may contribute both positively and negatively to outcomes. The use of the administrative database allowed us to review the outcomes of over 5000 infants for our study. We found that infants with hydrocephalus had a higher treatment failure rate when they had undergone ETV compared with shunt placement, even when controlling for prematurity, IVH, 
and myelomeningocele, which affected the success of both shunts and ETVs.

Although prior studies in Uganda did show improved outcomes with the addition of CPC to ETV, those populations did not include a significant number of infants born prematurely or infants with posthemorrhagic hydrocephalus. Further analysis of this additional surgical procedure in North American patients is needed.

Future studies should evaluate which physiological changes may be present that improve survival of both shunts and ETVs that are performed after 90 days of life compared with those performed within the first 90 days of life.

\section{Disclosure}

The authors report no conflict of interest concerning the materials or methods used in this study or the findings specified in this paper.

Author contributions to the study and manuscript preparation include the following. Conception and design: Goumnerova, Jernigan. Acquisition of data: Jernigan, Graham. Analysis and interpretation of data: all authors. Drafting the article: Jernigan. Critically revising the article: all authors. Reviewed submitted version of manuscript: all authors.

\section{References}

1. Berry JG, Hall MA, Sharma V, Goumnerova L, Slonim AD, Shah SS: A multi-institutional, 5-year analysis of initial and multiple ventricular shunt revisions in children. Neurosurgery 62:445-454, 2008

2. Berry JG, Lieu TA, Forbes PW, Goldmann DA: Hospital volumes for common pediatric specialty operations. Arch Pediatr Adolesc Med 161:38-43, 2007

3. Caldarelli M, Di Rocco C, La Marca F: Shunt complications in the first postoperative year in children with meningomyelocele. Childs Nerv Syst 12:748-754, 1996

4. Choudhury AR: Avoidable factors that contribute to the complications of ventriculoperitoneal shunt in childhood hydrocephalus. Childs Nerv Syst 6:346-349, 1990

5. Cochrane DD, Kestle JR: The influence of surgical operative experience on the duration of first ventriculoperitoneal shunt function and infection. Pediatr Neurosurg 38:295-301, 2003

6. Cochrane DD, Kestle J: Ventricular shunting for hydrocephalus in children: patients, procedures, surgeons and institutions in English Canada, 1989-2001. Eur J Pediatr Surg 12 Suppl 1:S6-S11, 2002

7. Egger D, Balmer B, Altermatt S, Meuli M: Third ventriculostomy in a single pediatric surgical unit. Childs Nerv Syst 26:93-99, 2010

8. Faggin R, Bernardo A, Stieg P, Perilongo G, d'Avella D: Hydrocephalus in infants less than six months of age: effectiveness of endoscopic third ventriculostomy. Eur J Pediatr Surg 19:216-219, 2009

9. Fritsch MJ, Kienke S, Ankermann T, Padoin M, Mehdorn HM: Endoscopic third ventriculostomy in infants. J Neurosurg 103 (1 Suppl):50-53, 2005

10. Goumnerova LC, Frim DM: Treatment of hydrocephalus with third ventriculocisternostomy: outcome and CSF flow patterns. Pediatr Neurosurg 27:149-152, 1997

11. Jenkins KJ, Newburger JW, Lock JE, Davis RB, Coffman GA, Iezzoni LI: In-hospital mortality for surgical repair of congenital heart defects: preliminary observations of variation by hospital caseload. Pediatrics 95:323-330, 1995

12. Kelley ET, Arispe I, Holmes J: Beyond the initial indicators: lessons from the OECD Health Care Quality Indicators Proj- ect and the US National Healthcare Quality Report. Int J Qual Health Care 18 (Suppl 1):45-51, 2006

13. Klimo P Jr, Thompson CJ, Drake J, Kestle JR: Assessing the validity of the endoscopic shunt insertion trial: did surgical experience affect the results? J Neurosurg 101 (2 Suppl): 130-133, 2004

14. Kulkarni AV, Drake JM, Mallucci CL, Sgouros S, Roth J, Constantini S, et al: Endoscopic third ventriculostomy in the treatment of childhood hydrocephalus. J Pediatr 155:254259, 2009

15. Patwardhan RV, Nanda A: Implanted ventricular shunts in the United States: the billion-dollar-a-year cost of hydrocephalus treatment. Neurosurgery 56:139-145, 2005

16. Piatt JH Jr, Carlson CV: A search for determinants of cerebrospinal fluid shunt survival: retrospective analysis of a 14-year institutional experience. Pediatr Neurosurg 19:233-242, 1993

17. Sainte-Rose C, Piatt JH, Renier D, Pierre-Kahn A, Hirsch JF, Hoffman HJ, et al: Mechanical complications in shunts. Pediatr Neurosurg 17:2-9, 1991-1992

18. Shah SS, Hall M, Slonim AD, Hornig GW, Berry JG, Sharma $\mathrm{V}$ : A multicenter study of factors influencing cerebrospinal fluid shunt survival in infants and children. Neurosurgery 62:1095-1103, 2008

19. Simon T, Riva-Cambrin J, Srivastava R, Bratton SL, Dean JM, Kestle JR, et al: Hospital care for children with hydrocephalus in the United States: utilization, charges, comorbidities, and deaths. J Neurosurg Pediatr 1:131-137, 2008

20. Smink DS, Fishman SJ, Kleinman K, Finkelstein JA: Effects of race, insurance status, and hospital volume on perforated appendicitis in children. Pediatrics 115:920-925, 2005

21. Smith ER, Butler WE, Barker FG II: In-hospital mortality rates after ventriculoperitoneal shunt procedures in the United States, 1998 to 2000: relation to hospital and surgeon volume of care. J Neurosurg 100 (2 Suppl Pediatrics):90-97, 2004

22. Stein SC, Guo W: Have we made progress in preventing shunt failure? A critical analysis. J Neurosurg Pediatr 1:40-47, 2008

23. Stein SC, Guo W: A mathematical model of survival in a newly inserted ventricular shunt. J Neurosurg 107 (6 Suppl): 448-454, 2007

24. Tuli S, Drake J, Lawless J, Wigg M, Lamberti-Pasculli M: Risk factors for repeated cerebrospinal shunt failures in pediatric patients with hydrocephalus. J Neurosurg 92:31-38, 2000

25. Vitale MA, Arons RR, Hyman JE, Skaggs DL, Roye DP, Vitale MG: The contribution of hospital volume, payer status, and other factors on the surgical outcomes of scoliosis patients: a review of 3,606 cases in the State of California. J Pediatr Orthop 25:393-399, 2005

26. Wagner W, Koch D: Mechanisms of failure after endoscopic third ventriculostomy in young infants. J Neurosurg 103 (1 Suppl):43-49, 2005

27. Warf BC: Comparison of endoscopic third ventriculostomy alone and combined with choroid plexus cauterization in infants younger than 1 year of age: a prospective study in 550 African children. J Neurosurg 103 (6 Suppl):475-481, 2005

\footnotetext{
Manuscript submitted March 22, 2013.

Accepted November 4, 2013.

Portions of this work were presented in abstract form as proceedings at the AANS Pediatric Section Meeting, Cleveland, $\mathrm{OH}$, November 2010.

Please include this information when citing this paper: published online January 3, 2014; DOI: 10.3171/2013.11.PEDS13138.

Address correspondence to: Liliana Goumnerova, M.D., Department of Neurosurgery, Boston Children's Hospital, Hunnewell 2,300 Longwood Ave., Boston, MA 02115. email: Liliana.Goumnerova @ childrens.harvard.edu.
} 